\title{
Tissue spatial correlation as cancer marker
}

Masanori Takabayashi

Hassaan Majeed

Andre Kajdacsy-Balla

Gabriel Popescu 


\title{
Tissue spatial correlation as cancer marker
}

\author{
Masanori Takabayashi, ${ }^{a, b, *}$ Hassaan Majeed, ${ }^{c}$ Andre Kajdacsy-Balla, ${ }^{d}$ and Gabriel Popescu ${ }^{b, c}$ \\ ${ }^{a}$ Kyushu Institute of Technology, Department of Systems Design and Informatics, lizuka, Fukuoka, Japan \\ bUniversity of Illinois at Urbana-Champaign, Beckman Institute of Advanced Science and Technology, \\ Department of Electrical and Computer Engineering, Urbana, Illinois, United States \\ cUniversity of Illinois at Urbana-Champaign, Beckman Institute of Advanced Science and Technology, \\ Department of Bioengineering, Urbana, Illinois, United States \\ dUniversity of Illinois at Chicago, Department of Pathology, Chicago, Illinois, United States
}

\begin{abstract}
We propose an intrinsic cancer marker in fixed tissue biopsy slides, which is based on the local spatial autocorrelation length obtained from quantitative phase images. The spatial autocorrelation length in a small region of the tissue phase image is sensitive to the nanoscale cellular morphological alterations and can hence inform on carcinogenesis. Therefore, this metric can potentially be used as an intrinsic cancer marker in histopathology. Typically, these correlation length maps are calculated by computing two-dimensional Fourier transforms over image subregions-requiring long computational times. We propose a more time-efficient method of computing the correlation map and demonstrate its value for diagnosis of benign and malignant breast tissues. Our methodology is based on highly sensitive quantitative phase imaging data obtained by spatial light interference microscopy. ๑ The Authors. Published by SPIE under a Creative Commons Attribution 3.0 Unported License. Distribution or reproduction of this work in whole or in part requires full attribution of the original publication, including its DOI. [DOI: 10.1117/1.JBO.24.1.016502]
\end{abstract}

Keywords: quantitative phase imaging; biopsy slide diagnosis; breast cancer.

Paper 180327R received Jun. 5, 2018; accepted for publication Dec. 28, 2018; published online Jan. 21, 2019.

\section{Introduction}

According to the World Health Organization, cancer is a major cause of death globally. ${ }^{1}$ Effective treatment strategies require early and accurate diagnosis of the disease. The gold standard method for cancer diagnosis is the microscopic investigation of a stained tissue biopsy by a trained clinical pathologist. Through this investigation, the pathologist looks for morphological signatures of either normal or abnormal tissue. Being qualitative, this type of assessment leads not only to interobserver discrepancy but also to automation of some or part of the process through machine learning where image analysis is complicated by stain variability. ${ }^{2,3}$ Ensuring consistency in the disease signatures extracted through image analysis of stained tissue remains a significant challenge due to variations in tissue preparation. $^{4}$

Quantitative phase imaging $(\mathrm{QPI})^{5}$ is a label-free microscopy technique where contrast is generated by the optical path-length difference (OPD), which is the product of the local thickness and refractive index of the specimen. ${ }^{5-7}$ For a thin specimen, such as a tissue histology, the thickness can be considered spatially invariant, in which case QPI images are proportional to a mean refractive index map, ${ }^{8,9}$ i.e., a refractive index map integrated along the $z$ axis. Since the refractive index is proportional to the dry mass content of cells and cellular matrix, it informs on tissue density as well as cell organization within tissue. ${ }^{10,11}$ Tissue refractive index-based markers have been used in the past for medical diagnosis and prognosis of several types of cancers and diseases. ${ }^{12-24}$ By generating contrast, label-free QPI lends itself much more readily to automated image analysis than bright-field microscopy, since stain variation is no longer an issue. ${ }^{13}$

In addition to the advantages of label-free imaging, the contrast mechanism in QPI provides access to additional markers of

*Address all correspondence to Masanori Takabayashi, E-mail: takabayashi@ ces.kyutech.ac.jp disease, which are of value to histopathology. ${ }^{14,25}$ In particular, since QPI systems employ interferometric measurements, they are sensitive to subwavelength fluctuations in OPD in both space and time. ${ }^{5}$ Therefore, local fluctuations in quantitative phase images inform on nanoscale morphological alterations of cell structures, due to the dry mass accumulation as well changes in extracellular matrix components. The tissue metric referred to as "disorder strength," a marker of the spatial fluctuations of refractive index, i.e., nanoscale morphological alterations, was first used as a marker for pancreatic cancer diagnosis by Subramanian et al. ${ }^{26}$ Their group used a spectroscopic imaging modality to measure this marker and have subsequently employed it in diagnostic studies related to prostate, colon, breast, lung, and other cancers. ${ }^{27-36}$ Thereafter, Eldridge et al. ${ }^{37}$ successfully extracted the disorder strength from quantitative phase images and demonstrated the relationship of the marker to cancer cell mechanical properties. They applied this analysis to colon, skin, and lung cancer cells to demonstrate an inverse relationship between shear stiffness and disorder strength. Building on these results, Muñoz et al. ${ }^{38}$ used QPI to study the on-set and progression of shear stiffness changes during malignant transformation in bronchial epithelial cells. Our group also showed that the disorder strength measured by spatial light interference microscopy (SLIM), a sensitive white light QPI method, is a quantitative marker of malignancy that can be used to classify benign and malignant breast tissue microarray (TMA) cores.

In this paper, we propose the local spatial autocorrelation length as an intrinsic marker of nanoscale morphological alteration in fixed tissue biopsies. Since the spatial autocorrelation length is related to the spatial refractive index fluctuations, which has been shown to detect malignancy in previous works, the length in a local region of tissue can be correlated with carcinogenesis. In the past, the local spatial autocorrelation length map was computed by calculating the two-dimensional (2-D) correlation function over regions of an image leading to long computation times. In this work, we present a more 
efficient algorithm for calculating the local spatial autocorrelation length map, requiring a smaller number of calculation steps. We then classify benign and malignant breast TMA cores using the local spatial autocorrelation length calculated by the proposed algorithm.

\section{Materials and Methods}

\subsection{Spatial Light Interference Microscopy}

The phase image, $\phi(x, y)$, measured in QPI is given by the equation:

$\phi(x, y)=\frac{2 \pi}{\lambda} \int_{0}^{L(x, y)} n(x, y, z) \mathrm{d} z$

where $n(x, y, z)$ is the refractive index contrast between the tissue and the surrounding medium, $L(x, y)$ is the thickness of the tissue, and $\lambda$ is the illumination wavelength. Here, we note that the intratissue refractive index fluctuations are integrated along $z$ direction. In this work, we use $4-\mu$ m-thick tissue slices with small lateral variation in thickness $[L(x, y) \approx L]$ as the specimen.

A schematic of the SLIM setup is shown in Fig. 1(a). The SLIM module is attached to a commercial phase contrast microscope (PCM). The lamp filament is imaged onto the condenser annulus (Köhler illumination conditions), which is located at the front focal plane of the condenser lens. The specimen is located at the back focal plane of the condenser lens, and front focal plane of the objective. The scattered and unscattered fields are relayed by the objective and tube lenses. As a result, the expanded phase contrast image that has the intensity distribution in accordance with the phase contrast caused by the specimen is observed at the image plane. However, because the output of PCM is qualitative, the phase image, $\phi(x, y)$, cannot be directly retrieved from this image. The SLIM module extracts $\phi(x, y)$ by

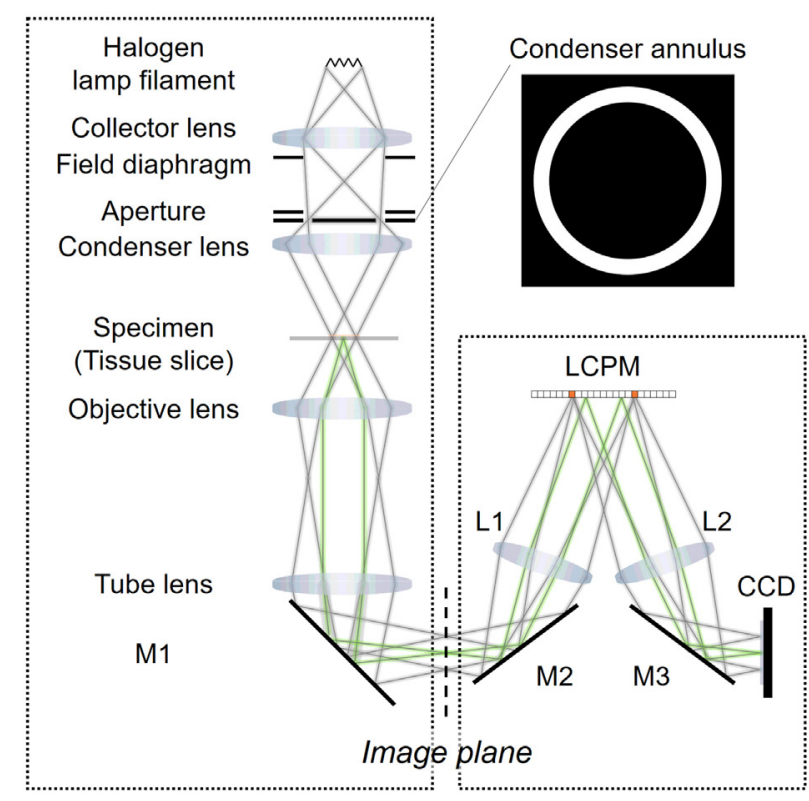

Commercial PCM
SLIM module (a)

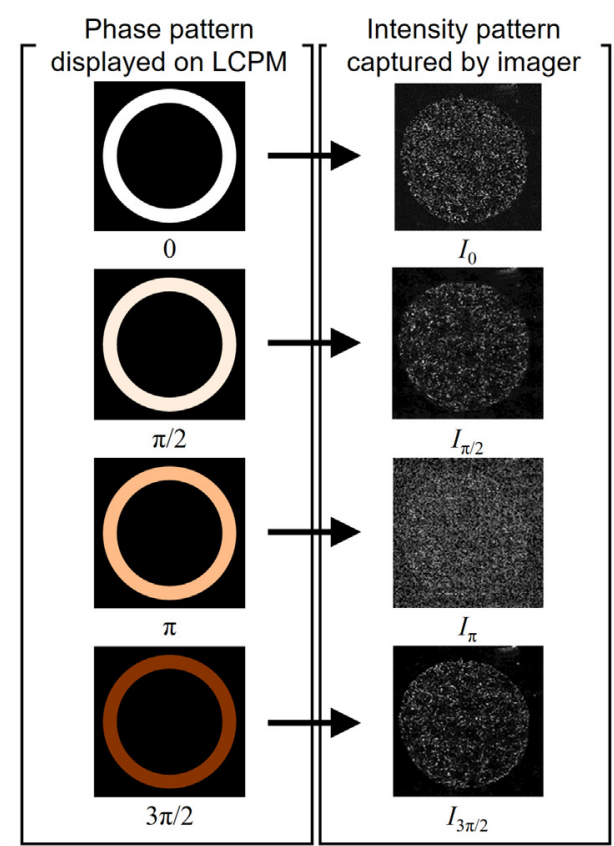

(b)
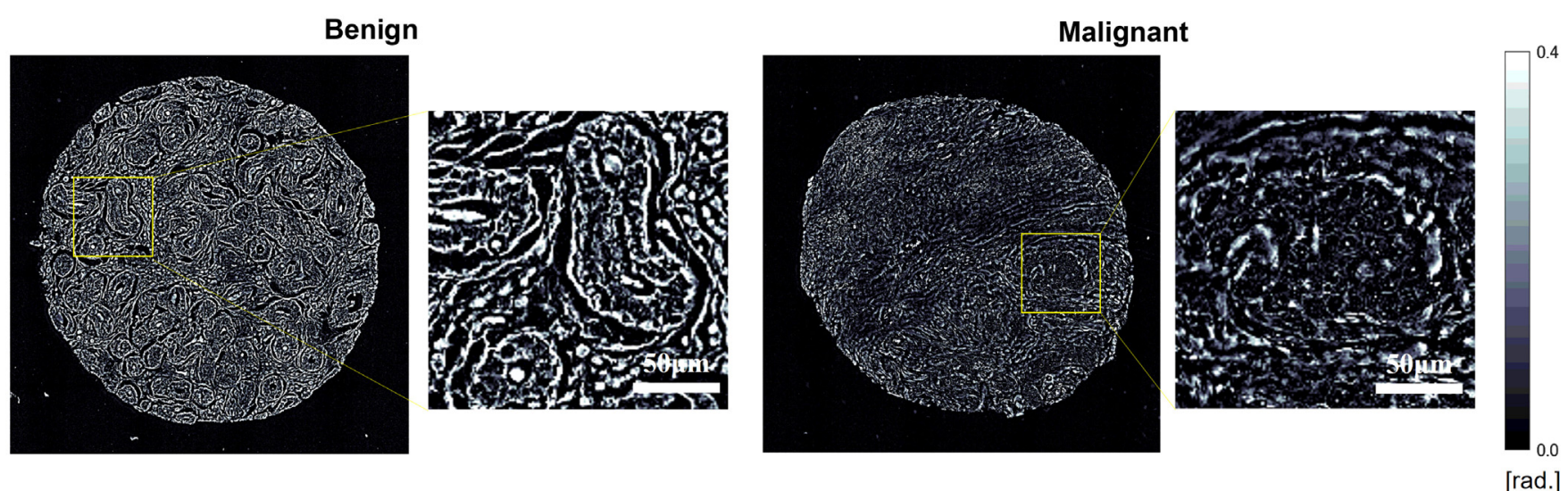

(c)

Fig. 1 SLIM system. (a) Optical setup. (b) Phase patterns displayed on LCPM and corresponding intensity patterns captured by CCD. (c) Example of quantitative phase images of benign and malignant breast tissue cores and their expanded views. 
phase modulating the incident light with respect to the scattered light. The field at the image plane is Fourier transformed by the lens L1, such that the unscattered light can be spatially isolated from the scattered light. Since the incident light has the ring form, by displaying the corresponding ring pattern on the reflective liquid crystal phase modulator (LCPM), we ensure that the scattered light remains unaffected. Four phase shifts are applied to the unscattered light at increments of $\pi / 2 \mathrm{rad}$, as shown in Fig. 1(b). The corresponding four images captured by the charge coupled device (CCD) are obtained. Consequently, the quantitative phase image is retrieved as described in Ref. 5. Figure 1(c) shows the quantitative phase image and its expanded view of benign and malignant breast tissue samples.

\subsection{Breast Tissue Microarrays}

The samples comprised a TMA of cores constructed from breast tissue biopsies of 400 different patients. Each biopsy was formalin fixed and paraffin embedded before sectioning it into slices of $4-\mu \mathrm{m}$ thickness each using a microtome. Two parallel, adjacent sections were selected from each biopsy, and one of these sections was stained using $\mathrm{H} \& \mathrm{E}$, leaving the other one unstained. Cores were then constructed for both the stained and unstained tissue, and these were mounted on separate slides after deparaffinization, using xylene as the mounting medium. The stained samples were imaged using a bright-field microscope, and their images were used by a board-certified pathologist for diagnosing each core. In this paper, we studied benign tissue as well as cancerous tissue from three different grades: benign $(N=20)$, malignant (grade $1, N=16)$, malignant (grade $2, N=16$ ), and malignant (grade $3, N=14$ ). Each patient consented to their tissue samples being used as part of the study, and the process of obtaining consent was approved by the Institute Review Board (IRB Protocol Number 20100519) at University of Illinois at Chicago (UIC). The data analysis was conducted on the samples at the University of Illinois at Urbana-Champaign (UIUC) after all patient identifiers had been removed. The procedures used in this study for conducting experiments using human subjects were also approved by the institute review board at UIUC (IRB Protocol Number 13900).

\subsection{Formulation for Local Spatial Autocorrelation Length Map}

As mentioned, the local spatial autocorrelation length depends on the morphological disorder, i.e., local refractive index fluctuations. When the refractive index is spatially disordered, the spatial autocorrelation length within the local area will shorten.
In general, the spatial autocorrelation length is calculated as the width of the spatial autocorrelation function. According to the Wiener-Khinchin theorem, the 2-D spatial autocorrelation function can be obtained by taking inverse 2-D Fourier transform of the spatial power spectrum. In other words, two 2-D Fourier transforms for each image, leading to long computation times. Thus, to avoid this problem, we propose a procedure that performs these calculation in the frequency-domain.

First, as shown in Fig. 2, we define the local spatial autocorrelation function as

$\Gamma\left(x, y ; x^{\prime}, y^{\prime}\right)=t\left(x, y ; x^{\prime}, y^{\prime}\right) \otimes_{x, y} t\left(x, y ; x^{\prime}, y^{\prime}\right)$,

where $\otimes_{x, y}$ denotes the 2-D correlation operation over $(x, y)$. Function $t\left(x, y ; x^{\prime}, y^{\prime}\right)$ is a local phase function centered at $\left(x^{\prime}, y^{\prime}\right)$ and is expressed as

$$
\begin{aligned}
t\left(x, y ; x^{\prime}, y^{\prime}\right)= & \phi(x, y) w\left(x, y ; x^{\prime}, y^{\prime}\right) \\
& -\left\langle\phi(x, y) w\left(x, y ; x^{\prime}, y^{\prime}\right)\right\rangle_{(x, y)},
\end{aligned}
$$

where $w\left(x, y ; x^{\prime}, y^{\prime}\right)=\operatorname{rect}\left(\frac{x-x^{\prime}}{a}\right) \operatorname{rect}\left(\frac{y-y^{\prime}}{a}\right)$ is a local window function centered at $\left(x^{\prime}, y^{\prime}\right)$, of width of $a$. The angular brackets denote averaging within the local window.

Next, we define the local spatial autocorrelation length map, $\rho\left(x^{\prime}, y^{\prime}\right)$, as the variance of the probability density, which can be obtained by normalizing $\Gamma\left(x, y ; x^{\prime}, y^{\prime}\right)$ by $\iint \Gamma\left(x, y ; x^{\prime}, y^{\prime}\right) \mathrm{d} x \mathrm{~d} y$ :

$\rho^{2}\left(x^{\prime}, y^{\prime}\right)=\frac{\iint\left(x^{2}+y^{2}\right) \Gamma\left(x, y ; x^{\prime}, y^{\prime}\right) \mathrm{d} x \mathrm{~d} y}{\iint \Gamma\left(x, y ; x^{\prime}, y^{\prime}\right) \mathrm{d} x \mathrm{~d} y}$.

Here, $\rho\left(x^{\prime}, y^{\prime}\right)$ can be related to the bandwidth map of the spatial power-spectrum, $\tau\left(x^{\prime}, y^{\prime}\right)$, as $\rho\left(x^{\prime}, y^{\prime}\right) \tau\left(x^{\prime}, y^{\prime}\right)=2 \pi$. The local bandwidth, $\tau\left(x^{\prime}, y^{\prime}\right)$, itself is defined as

$\tau^{2}\left(x^{\prime}, y^{\prime}\right)=\frac{\iint\left(k_{x}^{2}+k_{y}^{2}\right)\left|t\left(k_{x}, k_{y} ; x^{\prime}, y^{\prime}\right)\right|^{2} \mathrm{~d} k_{x} \mathrm{~d} k_{y}}{\iint\left|t\left(k_{x}, k_{y} ; x^{\prime}, y^{\prime}\right)\right|^{2} \mathrm{~d} k_{x} \mathrm{~d} k_{y}}$,

where $t\left(k_{x}, k_{y} ; x^{\prime}, y^{\prime}\right)$ is the Fourier transform of $t\left(x, y ; x^{\prime}, y^{\prime}\right)$ along $(x, y)$. Using the differentiation property of Fourier transforms as well as Parseval's theorem, this equation can be rewritten as

$\tau^{2}\left(x^{\prime}, y^{\prime}\right)=\frac{\iint\left[\left|\frac{\partial}{\partial x} t\left(x, y ; x^{\prime}, y^{\prime}\right)\right|^{2}+\left|\frac{\partial}{\partial y} t\left(x, y ; x^{\prime}, y^{\prime}\right)\right|^{2}\right] \mathrm{d} x \mathrm{~d} y}{\iint\left|t\left(x, y ; x^{\prime}, y^{\prime}\right)\right|^{2} \mathrm{~d} x \mathrm{~d} y}$.

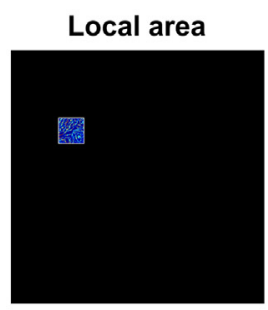

$t\left(x, y ; x^{\prime}, y^{\prime}\right)$

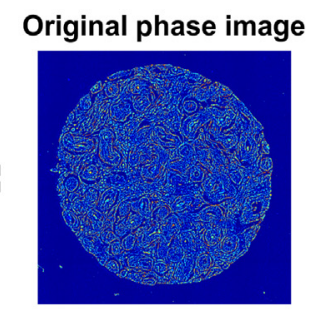

$\phi(x, y)$

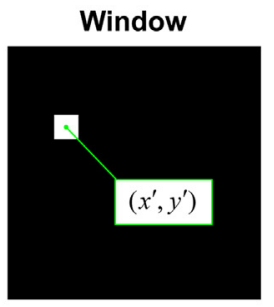

$w\left(x, y ; x^{\prime}, y^{\prime}\right)$

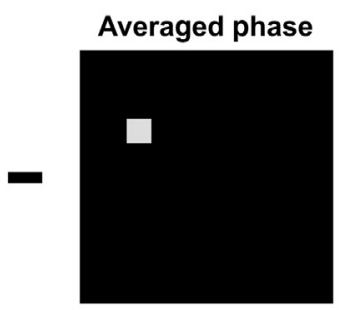

$\left\langle\phi(x, y) w\left(x, y ; x^{\prime}, y^{\prime}\right)\right\rangle_{(x, y)}$

Fig. 2 Definition of local 2-D function $t\left(x, y ; x^{\prime}, y^{\prime}\right)$. 

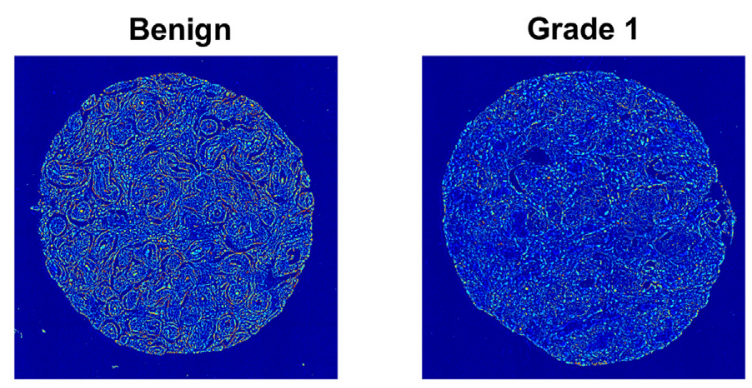

(a)
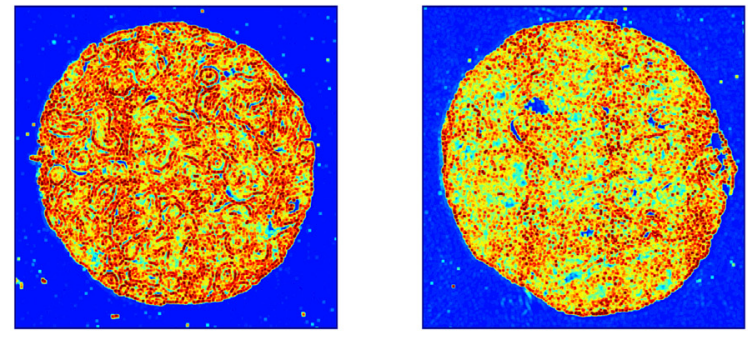

(b)
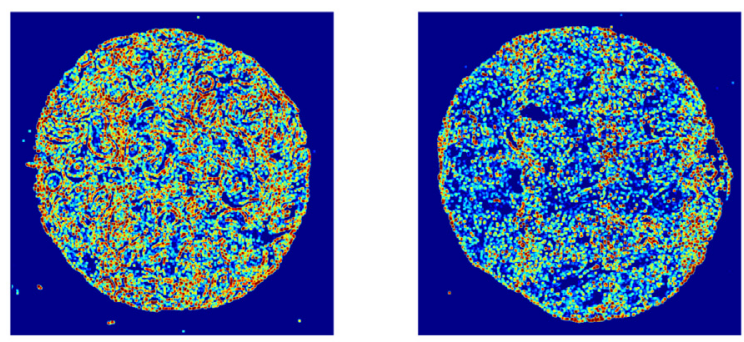
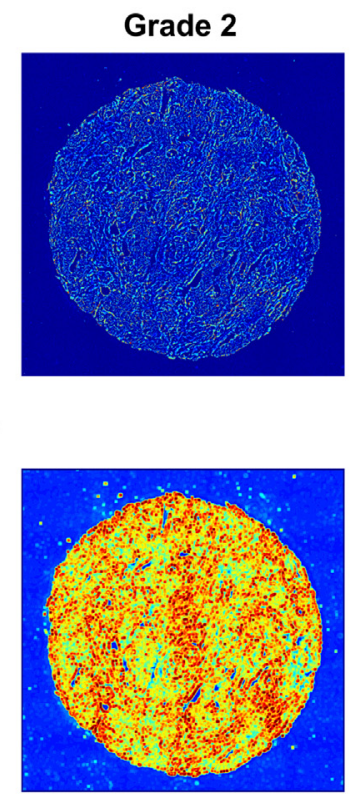

Grade 3
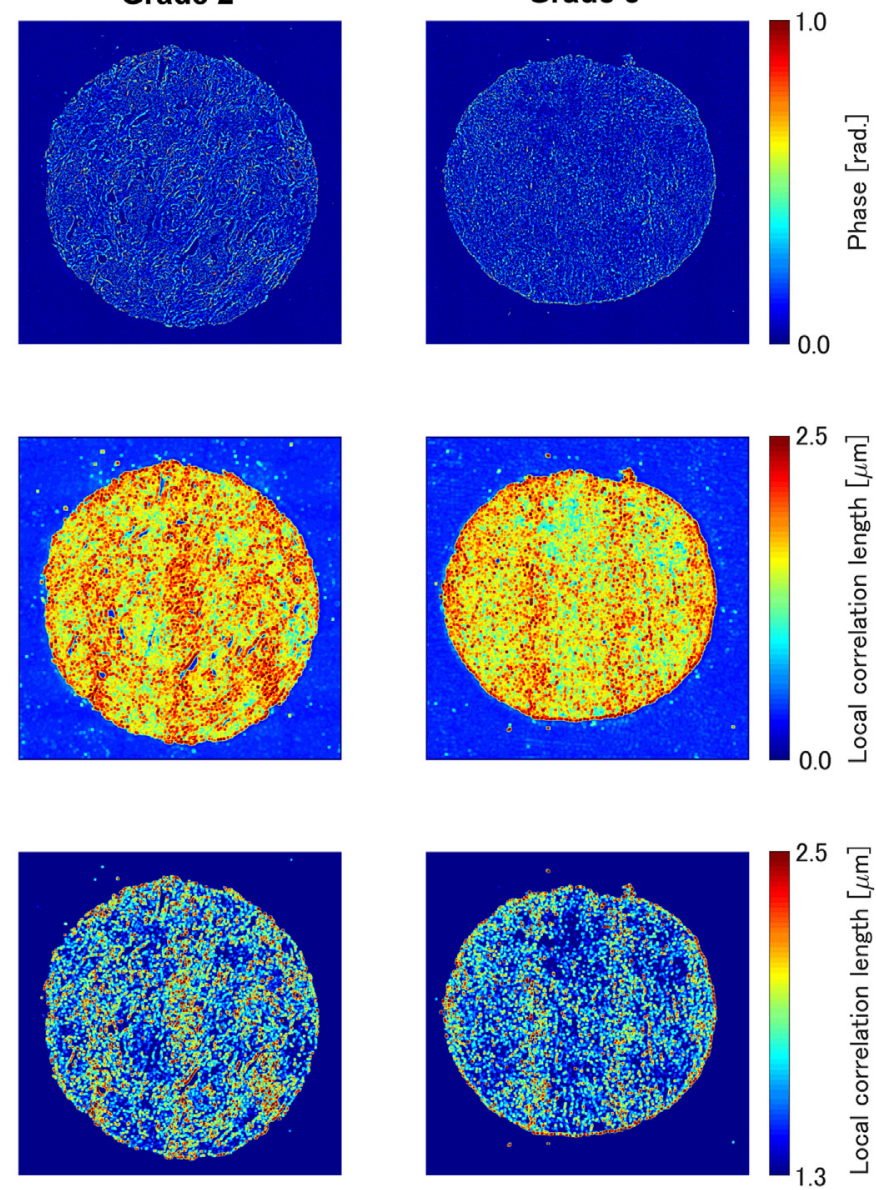

(c)

Fig. 3 Example of local spatial autocorrelation length maps. (a) Quantitative phase images, (b) the local correlation length maps, and (c) the local correlation length maps after applying the mask removing $\rho\left(x^{\prime}, y^{\prime}\right)<1.3 \mu \mathrm{m}$.

Finally, we can obtain the final result as

$$
\rho\left(x^{\prime}, y^{\prime}\right)=2 \pi \sqrt{\frac{\iint\left|t\left(x, y ; x^{\prime}, y^{\prime}\right)\right|^{2} \mathrm{~d} x \mathrm{~d} y}{\iint\left[\left|\frac{\partial}{\partial x} t\left(x, y ; x^{\prime}, y^{\prime}\right)\right|^{2}+\left|\frac{\partial}{\partial y} t\left(x, y ; x^{\prime}, y^{\prime}\right)\right|^{2}\right] \mathrm{d} x \mathrm{~d} y}} .
$$

Using Eq. (7), the local spatial autocorrelation length maps can be calculated as shown in Fig. 3(b), which were obtained from the phase maps of benign and malignant cores with different grades (grades 1, 2, and 3) as shown in Fig. 3(a). We used the local window with $a=64$ pixels $(8 \mu \mathrm{m})$.

\section{Results}

We demonstrate that malignant transformation is correlated with metrics calculated from local correlation maps. The dataset we analyzed consisted of 20 benign, 16 grade 1,16 grade 2 , and 14 grade 3 malignant cores. As the feature quantities, we use the value that is obtained by deviding the average by the standard deviation of the local correlation length. To extract only tissue regions, the background pixels were segmented out by setting a threshold in the $\rho(x, y)$ map. This threshold value was determined empirically, and all pixels having correlation lengths below $1.3 \mu \mathrm{m}$ were treated as background. The maps obtained after the background pixels reduction process are shown in Fig. 3(c). This calculation took $\sim 45 \mathrm{~min}$. per one core, which consists of $7040 \times 7040$ pixels $\left(880 \times 880 \mu \mathrm{m}^{2}\right)$ using PC with Intel Core i5-3470 CPU (3.20 GHz), 16.0 GB RAM, whereas it was estimated to take $90 \mathrm{~min}$. without our algorithm, i.e., with the algorithm using fast Fourier transform on the basis of the Wiener-Khinchin theorem. This calculation time can be improved, for example, by using GPU acceleration.

Figure 4 compares the average divided by the standard deviation of $\rho$ map between benign and malignant cores (grades 1,2 , and 3). The $p$-values that were obtained by two-sided Wilcoxon ranksum test are listed in Table 1. Since the $p$-value between 20 benign and 46 malignant cores was 0.000876 , the local correlation length correlates with cancer grades. Furthermore, the results indicate that the statistically significant differences between cores with $>2$ intergrade differences. This means that this label-free marker can be used for separating the lower risk cases (benign and grade 1) from the higher risk cases (grades 2 and 3). However, it may need to be combined with other markers for more detail separation of grades. We conclude that the local correlation map can potentially be 


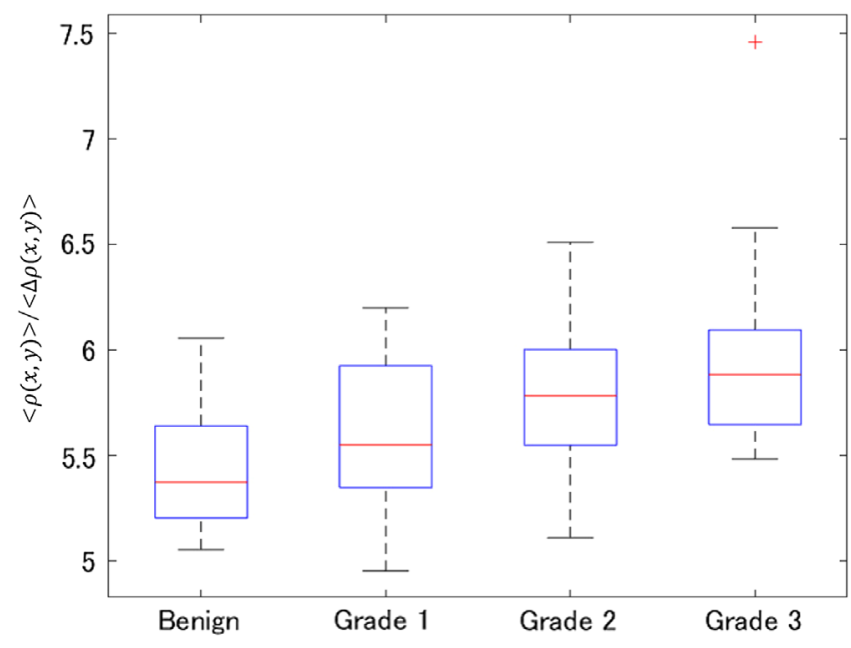

Fig. 4 Local spatial autocorrelation length of benign $(N=20)$ and grade $1(N=16)$, grade $2(N=16)$, and grade $3(N=14)$ tissues.

Table 1 The $p$-values between different grades.

\begin{tabular}{lc} 
Grades to be evaluated & $p$-value \\
\hline Benign-Malignant (G1, G2, G3) & 0.000876 \\
Benign and Malignant (G1)—Malignant (G2, G3) & 0.000335 \\
Benign—Malignant (G1) & 0.101101 \\
Benign-Malignant (G2) & 0.005891 \\
Benign-Malignant (G3) & 0.000498 \\
Malignant (G1)—Malignant (G2) & 0.193509 \\
Malignant (G1)—Malignant (G3) & 0.018837 \\
Malignant (G2)—Malignant (G3) & 0.417581 \\
\hline
\end{tabular}

used by clinical pathologists as a supplementary label-free disease marker for gauging the onset of malignancy especially in borderline cases.

\section{Summary and Conclusion}

We have presented an efficient algorithm for the computation of the local correlation length within refractive index maps of fixed breast tissue biopsy slides. This length metric describes the local refractive index fluctuations within the tissue specimen. Since in this work the refractive index maps are extracted using SLIM, which has sub-nanometer optical path length sensitivity, the correlation length is indicative of nanoscale cellular morphology. Standard computation of correlation length maps involves 2-D Fourier transforms, which can lead to long computation times, especially for large analysis window sizes. We improve calculation throughput by performing part of the computation in the frequency domain.

A comparison of the extracted correlation lengths between benign and malignant TMA cores showed that this metric is on average smaller for malignant cores indicating increased randomization of tissue morphology (as captured by its refractive index). Statistically significant differences in correlation lengths were observed between the two classes ( $N=20$ for benign and $N=46$ for malignant) indicating that this label-free disease marker can potentially be used by clinical pathologists for gauging the onset of malignancy especially in borderline cases. Furthermore, although the separation between neighboring grades cannot be achieved without the help of other markers, the results indicated that the local correlation length can also be used for separating the lower risk cases (benign and grade 1) from the higher risk cases (grades 2 and 3).

On the other hand, there is room for improvement on the calculation time and the screening accuracy. For example, calculation acceleration by GPU will drastically improve the calculation time, and the optimization of local window size and combining with other markers such as disorder strength ${ }^{8}$ will contribute to improve the screening accuracy. Because SLIM can be implemented as an upgrade of the existing microscopes, the extraction of intrinsic markers from quantitative phase images obtained through SLIM is expect to be plugged into the existing pathology work flow. Tissue spatial correlation information can add to the existing toolbox that the pathologists already have and help improve diagnosis accuracy and objectivity.

\section{Disclosures}

Gabriel Popescu has financial interest in Phi Optics, Inc., a company developing QPI technology for materials and life science applications. The remaining authors declare no competing financial interests.

\section{Acknowledgments}

We would like to thank Prof. Garth H. Rauscher for his pathology expertise. This work was supported by National Science Foundation (CBET-0939511 STC, DBI 1450962 EAGER, IIP-1353368, and CBET-1040461 MRI) and by JSPS KAKENHI Grant No. 18K14150. This work was based partly on scientific content previously reported in SPIE proceedings: M. Takabayashi, H. Majeed, A. Kajdacsy-Balla, and G. Popescu, "High throughput calculation of local spatial autocorrelation length for label-free diagnosis of tissue biopsy", Proc. SPIE 10503, Quantitative Phase Imaging IV, 105032B; doi: https://doi.org/10.1117/12.2293991.

\section{References}

1. "World health organization fact sheets: cancer," http://www.who.int/ news-room/fact-sheets/detail/cancer (12 September 2018).

2. M. McLean et al., "Interobserver variation in prostate cancer gleason scoring: are there implications for the design of clinical trials and treatment strategies?" Clin. Oncol. 9, 222-225 (1997).

3. A. R. Feinstein, N. A. Gelfman, and R. Yesner, "Observer variability in the histopathologic diagnosis of lung cancer," Am. Rev. Resp. Dis. 101, 671-684 (1970).

4. A. Madabuhushi and G. Lee, "Image analysis and machine learning in digital pathology: challenges and opportunities," Med. Image Anal. 33, 170-175 (2016).

5. G. Popescu, Quantitative Phase Imaging of Cells and Tissues, McGraw Hill, New York (2011).

6. Z. Wang et al., "Spatial light interference microscopy (slim)," Opt. Express 19, 1016-1026 (2011).

7. P. Wang et al., "Spatial-domain low-coherence quantitative phase microscopy for cancer diagnosis," Opt. Lett. 35, 2840-2842 (2010).

8. M. Takabayashi et al., "Disorder strength measured by quantitative phase imaging as intrinsic cancer marker in fixed tissue biopsies," PLoS One 13, e0194320 (2018). 
9. M. E. Kandel et al., "Label-free tissue scanner for colorectal cancer screening," J. Biomed. Opt. 22, 066016 (2017).

10. M. Mir et al., "Optical measurement of cycle-dependent cell growth," Proc. Natl. Acad. Sci. 108, 13124-13129 (2011).

11. K. L. Cooper et al., "Multiple phases of chondrocyte enlargement underlie differences in skeletal proportions," Nature 495, 375-378 (2013).

12. H. Majeed et al., "Breast cancer diagnosis using spatial light interference microscopy," J. Biomed. Opt. 20, 111210 (2015).

13. M. Mir et al., "Label-free quantitative evaluation of breast tissue using spatial light interference microscopy (slim)," Sci. Rep. 8, 6875 (2018).

14. H. Majeed et al., "Quantifying collagen fiber orientation in breast cancer using quantitative phase imaging," J. Biomed. Opt. 22, 046004 (2017).

15. H. Majeed et al., "Quantitative phase imaging for medical diagnosis," J. Biophotonics 10, 177-205 (2017).

16. T. H. Nguyen et al., "Automatic gleason grading of prostate cancer using quantitative phase imaging and machine learning," J. Biomed. Opt. 22, 036015 (2017).

17. S. Ban et al., "Optical properties of acute kidney injury measured by quantitative phase imaging," Biomed. Opt. Express 9, 921-932 (2018).

18. Y. Park et al., "Refractive index maps and membrane dynamics of human red blood cells parasitized by plasmodium falciparum," Proc. Natl. Acad. Sci. U. S. A. 105, 13730-13735 (2008).

19. S. Sridharan et al., "Prediction of prostate cancer recurrence using quantitative phase imaging," Sci. Rep. 5, 9976 (2015).

20. Z. Wang et al., "Tissue refractive index as marker of disease," J. Biomed. Opt. 16, 116017 (2011).

21. R. K. Bista et al., "Nuclear nano-morphology markers of histologically normal cells detect the "field effect" of breast cancer," Breast Cancer Res. Treat. 135, 115-124 (2012).

22. Y. Liu et al., "Investigation of nanoscale structural alterations of cell nucleus as an early sign of cancer," BMC Biophys. 7, 1 (2014).

23. P. Wang et al., "Nanoscale nuclear architecture for cancer diagnosis beyond pathology via spatial-domain low-coherence quantitative phase microscopy," J. Biomed. Opt. 15, 066028 (2010).

24. H. V. Pham, L. Pantanowitz, and Y. Liu, "Quantitative phase imaging to improve the diagnostic accuracy of urine cytology," Cancer Cytopathol. 124, 641-650 (2016).

25. S. Sridharan et al., "Prediction of prostate cancer recurrence using quantitative phase imaging," Sci. Rep. 6, 33818 (2016).

26. H. Subramanian et al., "Partial-wave microscopic spectroscopy detects subwavelength refractive index fluctuations: an application to cancer diagnosis," Opt. Lett. 34, 518-520 (2009).

27. J. Chandler et al., "High-speed spectral nanocytology for early cancer screening," J. Biomed. Opt. 18, 117002 (2013).

28. D. Damania et al., "Role of cytoskeleton in controlling the disorder strength of cellular nanoscale architecture," Biophys. J. 99, 989-996 (2010).

29. D. Damania et al., "Nanocytology of rectal colonocytes to assess risk of colon cancer based on field cancerization," Cancer Res. 72, 2720-2727 (2012).
30. J. S. Kim et al., "The influence of chromosome density variations on the increase in nuclear disorder strength in carcinogenesis," Phys. Biol. 8, 015004 (2011).

31. V. J. Konda et al., "Nanoscale markers of esophageal field carcinogenesis: potential implications for esophageal cancer screening," Endoscopy 45, 983-988 (2013).

32. H. K. Roy et al., "Optical detection of buccal epithelial nanoarchitectural alterations in patients harboring lung cancer: Implications for screening," Cancer Res. 70, 7748-7754 (2010).

33. H. K. Roy et al., "Nano-architectural alterations in mucus layer fecal colonocytes in field carcinogenesis: potential for screening," Cancer Prev. Res. 6, 1111-1119 (2013).

34. H. K. Roy et al., "Nanocytological field carcinogenesis detection to mitigate overdiagnosis of prostate cancer: a proof of concept study," PLoS One 10, e0115999 (2015).

35. Y. E. Stypula-Cyrus et al., "HDAC up-regulation in early colon field carcinogenesis is involved in cell tumorigenicity through regulation of chromatin structure," PLoS One 8, e64600 (2013).

36. H. Subramanian et al., "Nanoscale cellular changes in field carcinogenesis detected by partial wave spectroscopy," Cancer Res. 69, 5357-5363 (2009).

37. W. J. Eldridge et al., "Optical phase measurements of disorder strength link microstructure to cell stiffness," Biophys. J. 112, 692-702 (2017).

38. A. Muñoz et al., "Cellular shear stiffness reflects progression of arsenicinduced transformation during g1," Carcinogenesis 39, 109-117 (2018).

Masanori Takabayashi is an associate professor at Kyushu Institute of Technology. He received his $\mathrm{BE}, \mathrm{ME}$, and $\mathrm{PhD}$ degrees in information science and technology from Hokkaido University in 2007, 2009, and 2012, respectively. His current research interests include volume holography, digital holography, and quantitative phase imaging for medical diagnosis.

Hassaan Majeed obtained his $\mathrm{PhD}$ in bioengineering at the University of Illinois at Urbana-Champaign. His thesis research was focused on using quantitative phase imaging for developing new methods for breast cancer diagnosis as well as looking for indicators of prognosis.

Andre Kajdacsy-Balla is a professor and director of the Transdisciplinary Pathology Division at the University of Illinois at Chicago. His clinical focus is anatomic pathology with special interest in gynecologic pathology and prostate cancer. His research interests are in the areas of TMAs, application of molecular techniques to tissue pathology, prostate cancer clinical outcomes prediction methods, and the effect of environmental agents on prostate cancer progression and metastasis.

Gabriel Popescu is a professor in electrical and computer engineering at UIUC. His research is focused on biomedical optics and quantitative phase imaging of cells and tissue. He founded Phi Optics, Inc., a startup company that commercializes quantitative phase imaging technology. He is a fellow of OSA and SPIE. 